\title{
Environmental Influences on Genomic Imprinting
}

\author{
Maya Kappil $^{1} \cdot$ Luca Lambertini $^{1,2} \cdot$ Jia Chen $^{1,3,4,5}$
}

Published online: 1 May 2015

(C) Springer International Publishing AG 2015

\begin{abstract}
Genomic imprinting refers to the epigenetic mechanism that results in the mono-allelic expression of a subset of genes in a parent-of-origin manner. These haploid genes are highly active in the placenta and are functionally implicated in the appropriate development of the fetus. Furthermore, the epigenetic marks regulating imprinted expression patterns are established early in development. These characteristics make genomic imprinting a potentially useful biomarker for environmental insults, especially during the in utero or early development stages, and for health outcomes later in life. Herein, we critically review the current literature regarding
\end{abstract}

This article is part of the Topical Collection on Environmental Epigenetics

\section{Jia Chen}

jia.chen@mssm.edu

Maya Kappil

maya.kappil@mssm.edu

Luca Lambertini

luca.lambertini@mssm.edu

1 Department of Preventive Medicine, Icahn School of Medicine at Mount Sinai, Box 1057, 1 Gustave Levy Place, New York, NY 10029, USA

2 Department of Obstetrics, Gynecology and Reproductive Science, Icahn School of Medicine at Mount Sinai, Box 1057, 1 Gustave Levy Place, New York, NY 10029, USA

3 Department of Pediatrics, Icahn School of Medicine at Mount Sinai, Box 1057, 1 Gustave Levy Place, New York, NY 10029, USA

4 Department of Oncological Sciences, Icahn School of Medicine at Mount Sinai, Box 1057, 1 Gustave Levy Place, New York, NY 10029, USA

5 Department of Medicine, Hematology, and Medical Oncology, Icahn School of Medicine at Mount Sinai, Box 1057, 1 Gustave Levy Place, New York, NY 10029, USA environmental influences on imprinted genes and summarize findings that suggest that imprinted loci are sensitive to known teratogenic agents, such as alcohol and tobacco, as well as less established factors with the potential to manipulate the in utero environment, including assisted reproductive technology. Finally, we discuss the potential of genomic imprinting to serve as an environmental sensor during early development.

Keywords Genomic imprinting · Environmental exposures · Early development $\cdot$ Epigenetic regulation $\cdot$ Placenta

\section{Introduction}

\section{Evolutionary Development and Function of Genomic Imprinting}

Diploidy confers the genome with a protective mechanism in which aberrations in one gene copy can be rescued by the presence of the alternate copy. However, approximately $1 \%$ of the human protein-coding genome is imprinted, a state referring to mono-allelic expression of genes in a parent-of-origin manner [1]. The presence of such genes overrides the benefit of diploidy and establishes the necessity of both maternal and paternal contributions for a viable fetus [2, 3]. In humans, imprinted genes are largely organized in well-conserved clusters and predominately expressed in the placenta, an organ that facilitates viviparity between mother and fetus [4]. Hence, fetal development is primarily maternally dictated. As early studies showed that paternally expressed imprinted genes promote growth while maternally expressed genes inhibit growth, it has been suggested that the phenomenon of imprinting evolved as a means to balance the bias in parental contributions to fetal development. Based on this theory, a parental conflict arises in which paternally expressed imprinted genes reflect a paternal 
interest to increase fetal nutritional intake to maximize the offspring's fitness. This is countered by maternally expressed imprinted genes that reflect a maternal interest to conserve her own fitness and resources for future offspring [5, 6]. In this context, appropriate development requires a carefully regulated balance in the expression of these genes. Postnatally, imprinted genes continue to play a key role in development and are largely expressed in the brain, influencing suckling, neonatal behavior, appetite, and metabolism [7].

Perturbation of the established balance between maternally and paternally expressed imprinted genes results in severe deleterious effects on the development of the offspring. Total loss of a parental complement results in unviable embryos, as observed by the failure to thrive of either androgenetic or gynogenetic embryos [2, 3], while loss of imprinting at specific imprinted loci results in fetal growth syndromes. These loci are often organized to include both maternally and paternally imprinted genes. Hence, loss of imprinting at these regions often results in complementary syndromes with opposing phenotypes determined by the affected allele's parent-oforigin. For instance, depending on the direction of the imposed expression imbalance, aberrations in imprinted genes in the chromosomal region $11 \mathrm{p} 15$ can result in either Beckwith-Wiedemann syndrome (BWS), a developmental disorder with clinical features that includes pediatric overgrowth [8], or Silver-Russell syndrome (SRS), a disorder characterized by prenatal and postnatal undergrowth [9].

More subtle disruption of imprinted genes have also been linked to several chronic diseases, most notably metabolic disorders such as diabetes [10-12]. Various studies have shown associations between SNP variants in loci containing imprinted genes and type 1 and type 2 diabetes [10-12]. Similarly, altered methylation of the imprinted genes GNASAS and INS have been implicated with higher risk of coronary heart disease [13]. Imprinting aberrations have also been linked with an increased risk of carcinogenesis, a link that is likely explained by the fact that placentation shares many key processes involved in tumor development, including rapid growth and angiogenesis. SNP variants in imprinted genes and altered methylation patterns in imprint regulatory regions have been observed in association with basal cell carcinoma [11], breast cancer [11, 14, 15], colorectal cancer [16, 17], hepatocellular carcinoma [18, 19], leukemia [20], and ovarian cancer [21]. Finally, as postnatal expression of imprinting genes is predominately observed in the brain, deregulation of imprinted genes has also been implicated in neurobehavioral defects in infants, including handling and quality of movement scores, and psychiatric disorders in adults, such as schizophrenia [22, 23].

\section{Establishment and Dynamics of Genomic Imprinting}

The parent-of-origin-associated mono-allelic expression of imprinted genes is dictated by the establishment, maintenance, and interpretation of epigenetic imprint regulatory elements in specific regions of the genome known as imprinting control regions (ICRs) [24]. These epigenetic imprint regulatory elements include DNA methylation, histone modifications, and long non-coding RNAs (lncRNAs). While regulation is likely dictated by the action and interaction of all these various elements, DNA methylation at ICRs is the most commonly assessed epigenetic element due to its technical feasibility and is, therefore, the most widely studied marker of imprinting.

To date, various mechanisms utilized by ICRs to orchestrate the coordinated regulation of imprinting clusters have been reported. For example, the imprinting status of one gene can be leveraged to dictate imprinted expression of downstream genes. This is the case at the NNAT/BLCAP locus, where the close proximity of $N N A T$ to the alternative promoters of $B L C A P$ results in diverting transcription through the weaker BLCAP promoter when NNAT is transcribed [25]. Similarly, imprinted IncRNAs, such as KCNQ1OT1, have also been observed to regulate the imprinted expression of downstream genes [25]. Chromatin structural changes can also be utilized to coordinate the expression of an imprinting cluster. For example, the imprinted expression of IGF2 and $H 19$ are mediated by methylation patterns that dictate long-range interactions between enhancers and promoters. Here, mutually exclusive access to enhancer elements by the respective promoters is determined by the methylation status of CTCF binding sites [9]. Finally, adding an additional layer of complexity to imprinting regulation is the fact that DNA methylation at ICRs have also been observed to interact with specific histone modifications [26, 27].

Establishment of imprinting involves a highly unique and articulated set of molecular mechanisms. ICR allele-specific methylation patterns contributing to the allele-specific expression of imprinted genes in fact escape the first genome-wide epigenetic reprogramming wave of the DNA methylation state that occurs following fertilization. Global methylation marks, reflecting the methylation patterns of parental sperm and egg genomes, are erased and re-established during the first wave as the cells of the zygote differentiate into specific lineages. Parental imprint marks are protected from this event and eventually reconfigured as the embryo develops according to the specific imprinting profile of each somatic tissue. During the differentiation of primordial germ cells (PGCs), a second methylation reprogramming event takes place solely in the developing PGCs to establish germ cells with imprinting marks representative of the sex of the developing embryo (i.e., paternal imprints established in primordial sperm cells and maternal imprints established in primordial oocytes) [28, 1]. This re-establishment of imprints during gametogenesis spans the maturation of the gametes, a process that comes to completion by birth for sperm and following puberty for oocytes [29]. These findings, together with the studies that have indicated that the retained imprint marks in somatic tissues undergo re-modeling throughout the gestational period, 
highlight a previously unknown dynamic in utero state of both somatic and gametic methylation imprints [30, 31].

The highly regulated molecular mechanism necessary to preserve imprinted marks during the epigenetic reprogramming following zygote formation highlights a possible role of imprinting in epigenetic inheritance. This hypothesis is further supported by additional epigenetic events that take place during the first genome-wide DNA methylation reprogramming wave, including (1) differential protamine-to-histone exchange at ICRs in the paternal chromosome [32, 33], (2) timely regulated expression of imprinted lncRNAs [34, 35], and (3) allelespecific ICR transcription intended at protecting ICRs from DNA methylation [36]. Perturbations of these highly regulated mechanisms in the early developmental phases are likely to be detectable in most tissues and to have wide-ranging effects.

The dynamic characteristic of the in utero imprint state suggests that imprinting marks may be sensitive to environmental exposures during pregnancy. Imprinting may therefore be a powerful sensor of alterations of both epigenetic reprogramming at fertilization and fetoplacental development in pregnancy, affording the unique opportunity to assess imprinting as a potential biomarker of the in utero environment.

\section{Environmental Factors Impact Imprinting}

A number of studies have explored the impact of environmental exposures on imprinting during the periconceptional and gestational period in human and animal studies. The studies included in the current review were selected based on a PubMed query for each known imprinted gene listed in the GeneImprint database [37] in conjunction with the terms environmental exposure. As summarized in Table 1, the most widely studied exposures of interest include known teratogenic agents, such as alcohol and tobacco, and organic pollutants. In addition to these traditional exogenous exposures, environmental manipulations with suspected imprinting-specific consequences, such as the use of assisted reproductive technology, as well as maternal nutrition have also been investigated. The following sections outline the known associations between these early life exposures and identified aberrations in imprinting genes.

\section{Maternal Nutrition}

The state of maternal nutrition is a critical determinant of fetal development. As such, the impact of maternal nutrition has been widely explored across various model systems. Additionally, identifying relevant windows of susceptibility is a study component most frequently incorporated in studies focusing on maternal nutrition as the exposure of interest. The Dutch famine studies are among the most well-described epidemiologic studies addressing the role of dietary restriction across specific gestational periods on health outcomes of the offspring. In a study examining the effect of famine on methylation levels of 15 imprinted loci, lower levels of INSIGF

Table 1 Studies reporting on the impact of environmental agents on imprinted loci during development

\begin{tabular}{|c|c|c|c|c|c|}
\hline Environmental agent & Locus & Assessment & Biospecimen & Model & Reference \\
\hline \multirow[t]{5}{*}{ Alcohol } & Gabra5 & Expression & Embryo; brain & Mouse & Toso et al. 2006 [56] \\
\hline & $G A B R B 3$ & Expression & Embryonic stem cells & Human & Krishnamoorthy et al. 2011 [57] \\
\hline & $H 19$ & Methylation & Brain; sperm & Mouse & Stouder et al. 2011 [42] \\
\hline & Peg3 & Methylation & Sperm & Mouse & Liang et al. 2014 [43•] \\
\hline & $N D N$ & Expression & Neural progenitor cells & Human & Tyler and Allan 2014 [44] \\
\hline \multirow{3}{*}{$\begin{array}{l}\text { Assisted reproductive } \\
\text { treatment }\end{array}$} & $I G F 2$ & Methylation & Blood; buccal cells & Human & Hiura et al. 2012 [49] \\
\hline & $H 19$ and $M E S T$ & Methylation & Placenta & Human & Nelissen et al. 2013 [50] \\
\hline & $\begin{array}{l}\text { Kcnq1ot1, Mest, Peg3, Plagl1, } \\
\text { and Snrpn }\end{array}$ & Methylation; expression & Testes & Mouse & Xu et al. 2014 [51] \\
\hline \multirow[t]{3}{*}{ Bisphenol A } & Slc22a18 & Expression & Embryo & Mouse & Kang et al. 2011 [46] \\
\hline & $\operatorname{Igf} 2 r, \mathrm{Peg} 3$, and $\mathrm{H} 19$ & Methylation & Fetal germ cell & Mouse & Zhang et al. 2012 [58] \\
\hline & $\begin{array}{l}\text { Snrpn, Ube3a, Igf2, Kcnq1ot1, } \\
\quad \text { Cdkn1c, and Ascl2 }\end{array}$ & Methylation; expression & Embryo; placenta & Mouse & Susiarjo et al. 2013 [45•] \\
\hline \multirow[t]{9}{*}{ Maternal nutrition } & $A B C A 1, G N A S A S$, and $M E G 3$ & Methylation & Whole blood & Human & Tobi et al. 2009 [38] \\
\hline & Cdknlc & Methylation & Neurons & Mouse & Vucetic et al. 2010 [59] \\
\hline & $I G F 2$ & Methylation & Cord blood & Human & Ba et al. 2011 [60] \\
\hline & $I G F 2 / H 19$ and $I N S$ & Methylation; & Whole blood & Human & Tobi et al. 2012 [61] \\
\hline & $I G F 2$ and $P E G 3$ & genotyping & Cord blood & Human & Haggarty et al. 2013 [41] \\
\hline & $A B C A 1$ & Methylation & Placenta; cord blood & Human & Houde et al. 2013 [62] \\
\hline & PLAGL1, SGCE, DLK1/MEG3, & Methylation; expression & Erythrocytes; cord blood & Human & Hoyo et al. 2014 [63] \\
\hline & and $I G F 2 / H 19$ & Methylation & Adrenal & Sheep & Williams-Wyss et al. 2014 [39] \\
\hline & $\operatorname{Ig} f 2$ and $\operatorname{Ig} f r 2$ & Methylation; expression & & & \\
\hline \multirow[t]{2}{*}{ Tobacco } & $G F I 1$ & Methylation & Cord blood & Human & Joubert et al. 2012 [47] \\
\hline & $M E G 3$ & Methylation & Whole blood & Human & Markunas et al. 2014 [48] \\
\hline
\end{tabular}


methylation and higher levels of $A B C A 1$ and GNASAS methylation were observed among individuals exposed periconceptionally and during early pregnancy compared to their unexposed same-sex siblings. A significant sex interaction was observed with the effect on INSIGF restricted to men and the impact on GNASAS methylation more pronounced among women. Interestingly, lower levels of GNASAS methylation were observed in a comparison between individuals exposed to famine later in pregnancy compared to their unexposed same-sex siblings, contrary to the direction of the association observed in the group exposed early in pregnancy. These findings are especially striking as the impact on imprinting was observed up to 60 years after the exposure event, indicating the persistence of the induced alterations [38]. Animal studies have also suggested varying susceptibility due to diet depending on the targeted window of gestation. In a study focusing on the impact of dietary restriction on fetal adrenal activation in sheep, changes in adrenal $\operatorname{Ig} f 2$ and $\lg f 2 r$ expression were observed when the restriction spanned the periconceptional and preimplantation period, but no changes in the expression of these imprinted genes were observed when the exposure occurred solely in the preimplantation period [39].

In addition to overall food restriction, there is also an interest on the role of specific dietary components, with an emphasis on nutrient supplements known to be involved in methyl group metabolism, including folate, betaine, and vitamins B6 and B12 [40]. Contrary to the findings of studies focusing on overall food restriction, an epidemiologic study investigating the impact of folate intake on select imprinted loci observed higher methylation of $I G F 2$ and lower methylation of PEG3 when the folate intake was restricted to 12 weeks after conception. No association between folate supplementation and methylation of the selected loci were observed when the window of analysis was restricted to the periconceptional or early pregnancy period [41].

\section{Alcohol}

Alcohol is a known teratogen, with in utero exposure linked to fetal alcohol syndrome, a developmental disorder that includes behavioral and neurodevelopmental deficits. Several studies focusing on identifying exposure-susceptible genetic targets responsible for the associated developmental phenotypes have reported on the role of imprinted genes as potential mediators. These studies varied in the administration, dose, and window of the alcohol exposure. Still, a few trends have emerged. Methylation of the $H 19$ differentially methylated region (DMR) was observed to be hypomethylated in the F1 sperm of pregnant mice orally exposed to $0.5 \mathrm{mg} / \mathrm{kg}$ /day ethanol during gestational days 10-18 [42]. Interestingly, this DMR was also observed to be hypomethylated in the F0 sperm of male mice exposed intragastrically prior to mating
[43•]. Additionally, in a study where female mice were orally exposed to $7 \mathrm{~g} / \mathrm{kg} /$ day from 7 days prior to mating until 15 17 days after mating, $N d n$ expression was upregulated in the neural progenitor cells of the embryos [44]. $N d n$ expression was also upregulated in the F1 cerebral cortex when the male parent was exposed prior to mating [43•]. Some inconsistencies remain unresolved. In the cerebral cortex of F2 mice, alcohol exposure during the prenatal period was associated with a decrease in Snrpn methylation [43•]. However, in another study, no differential methylation of Snrpn was observed in the brains of the F2 mice following alcohol exposure during the gestational period [42].

\section{BPA}

Bisphenol A (BPA) is a ubiquitous plasticizer long suspected to be an endocrine-disrupting agent and among the most commonly investigated organic pollutants. As for other exposures, studies that have investigated the role of BPA exposure on imprinting during development differ in the administration, dose, and timing of exposure depending on the question of interest. Despite these differences, these studies reveal that the impact BPA exerts on imprinting and phenotype is temporally and spatially specific. In a study examining the impact of BPA exposure on imprinting in embryonic and placental tissues in a mouse model, a greater number of imprinting errors were observed in the placenta than in the embryonic body. Additionally, an impact on imprinting could be observed at lower doses in the placenta than the embryo, suggesting that placental tissues are more sensitive to exposures than the protected embryo. Further analysis also revealed pathological differences between BPA-exposed and BPA-unexposed placental tissues [45•]. The temporal window of exposure also determined the effect on imprinting. In a mouse model study where the window of exposure was set to 8.5-12.5 days post coitum, loss of imprinting (LOI) was observed in just 2 out of 39 analyzed genes, Slc22al8 and Rtl/Trllas, across various embryonic tissues [46]. Similarly, in Susiarjo et al.'s study, when exposure was set to embryonic days 5.5-12.5, no differential LOI was observed in any of the 5 genes analyzed, which included several genes analyzed in Kang et al.'s study. However, when exposure spanned from 2 weeks prior to mating until embryonic day 9.5, LOI was observed in several genes, including Snrpn, Igf2, and Kcnq1ot1, in embryonic and placental tissues [45•].

\section{Tobacco}

Similar to alcohol, tobacco is a known teratogen, and the impact of fetal tobacco exposure is of continual concern. While few studies have specifically sought out the impact of in utero tobacco exposure on imprinting aberrations, differential methylation levels in imprinted genes have been identified 
in epigenome-wide association studies. In a $450 \mathrm{~K}$ analysis conducted in the Norwegian Mother and Child Cohort Study (MoBa) to assess the impact of maternal smoking on cord blood methylation levels, multiple GFI1-related CpG sites were shown to be inversely associated with maternal cotinine levels [47]. Several of these sites were validated in a $450 \mathrm{~K}$ analysis conducted in the Norway Facial Clefts Study (NCL) where smoking history was self-reported. Additionally, infants of active smokers had significantly higher methylation levels of MEG3-related CpG sites measured in blood DNA than infants of non-smokers/passive/infrequent smokers [48]. The identified $M E G 3$ sites were also observed to be nominally significant in the MoBa study [47].

\section{Assisted Reproductive Technologies (ART)}

The use of assisted reproductive technologies, including in vitro fertilization (IVF) and intracytoplasmic sperm injection (ICSI), involves procedures such as ovarian stimulation and embryonic culture. As imprinting marks are set during this period, errors may be introduced as a result of these artificial manipulations. While studies have reported an increased incidence of imprinting disorders among individuals conceived through ART, it is still unclear whether the observed trends are related to the use of the ART or reflect other underlying features related to ART use and fetal abnormalities, including advanced parental age and subfertility. A number of studies have investigated the impact of ART on imprinting errors. A case study by Hiura et al. indicated that among BWS and SRS cases with known methylation aberrations at the DMRs typically associated with these disorders, the presence of errors at additional DMRs is more common among BWS and SRS cases conceived via ART compared to BWS and SRS cases conceived naturally [49].

While the manipulations involved are largely shared between IVF and ICSI treatments, it still remains to be addressed whether these treatments should be considered as distinct exposures. A case-control study assessing placental DMRs of infants conceived either naturally or through IVF/ICSI determined no significant differences in the methylation levels of the selected DMRs between IVF- and ICSI-conceived infants. Combining both treatments, lower levels of MEST and H19 methylation levels were observed among the ART cases compared to controls. Additionally, higher expression of $H 19$ was observed among the ART cases compared to controls. However, no difference in MEST expression levels was observed [50]. Contrary to these findings, a mouse model study did observe differences in testes gene expression levels between IVF and ICSI cases, including genes assessed in the human study. In the mouse model study, reduced expression of Kcnqo1t1, Mest, Peg3, Plagl1, and Snrpn was observed among ICSI cases compared to naturally conceived controls in the F1 generation. Additionally, higher levels of methylation were observed in Mest, Peg3, Plagl1, Snrpn, and KvDMR. Altered expression and methylation levels of several of these genes could still be detected in the F2 generations [51]. However, the overall conclusions that can be drawn from studies focusing on ART are often limited by the low number of available cases for analysis.

\section{Perspectives}

While the literature does support the possible reprogramming of imprinted marks during early development due to environmental exposures, several points will have to be addressed to clarify the role of genomic imprinting in the exposure-outcome paradigm. Key among these is a focus on more comprehensive assessments of imprinting loci. To date, most studies report analyses of a few imprinting loci at a time. Moreover, while 100 imprinted human genes have been identified and hundreds more are predicted, most published results focus on a small subset, primarily the well-described genes IGF2 and H19. Such limited scope is at least partially attributable to limitations in the methodologies available to assess imprinting. In terms of overall expression, assessment of imprinted genes requires highly quantitative assays (such as qPCR) as expression is tightly regulated; thus, array-based methodologies (such as microarrays) are less ideal for measuring imprinted genes since they lack the required sensitivity. Assessing allele-specificity, on the other hand, requires the difficult task of distinguishing differential expression between nearly identical parental alleles.

Currently, the conventional methodology to determine imprinting is through the assessment of ICR methylation levels. However, ICRs have been clearly defined for only a subset of the known imprint genes; the assessment of differential methylation at these sites likely reflects only one of multiple means of regulation; and as the differential levels of methylation observed at these sites often span only a few percentage points, the biological relevance of the reported findings remains unclear. In fact, few studies have been able to link DNA methylation levels at these sites with expression levels, highlighting a less straight-forward relationship between the role of imprint regulatory marks and their impact on the expression of these loci [52, 53].

Rather than using proxy measures, parent-of-origin specific mRNA levels can be determined using RT-qPCR methods [52]. These assays provide comprehensive measurements of LOI that are independent of imprinting mechanism. However, they are labor-intensive and rely on the presence of prevalent SNPs in the transcript that allow for sufficient numbers of informative heterozygotes to identify parent-of-origin, restricting the number of loci that can be investigated using this method.

The number of loci investigated is also influenced by the number of loci that has been identified thus far. 
Current genome-wide approaches to identify additional candidates include array-based technologies, transcriptome profiling, and bisulfite sequencing. However, identification of imprinted genes through such experimental efforts may be hampered due to the fact that mono-allelic expression of a gene may only be present in a specific isoform, tissue, or developmental window $[52,54]$.

These limitations can be overcome with computational methods to identify imprinted genes.

These are largely informed by genetic and epigenetic sequence features commonly associated with known imprinted genes $[28,55]$. However, few sequence motifs have been identified thus far, and while promising, to date, only few putative imprinted genes have been experimentally validated, indicating a propensity of current models to still generate a large number false positives.

As the technology for more high throughput assessments of expression and methylation becomes more refined, a more complete profiling that will include previously less represented imprinted genes as well as accounting for the likely interaction among these genes will be called for.

In addition to expanding the investigated loci, a greater focus needs to be exerted to further delineate the observed associations between environmental exposures and imprinting aberrations. This is most feasibly accomplished in studies utilizing animal models where exposure periods are well-defined, subsequent imprinting defects can be identified in target tissues, and the persistence of these defects across generations can be examined. However, drawing meaningful conclusions from the studies that have been conducted thus far has been hampered by the variability in exposure administration, timing, and dosage. Additional studies will need to be conducted to clarify the differences in results obtained due to these varying factors.

Finally, in studies that relate exposures to specific phenotypes, the relationship between the exposure and phenotype and the relationship between exposure and imprinting status are often evaluated separately. While positive associations from these separate constructs are used to infer that any existing association between exposure and phenotype is mediated by imprinted genes, such conclusions can only be drawn once more formal testing of the associations has been conducted. Hence, to further elucidate the biological mechanism underlying the pathway between exposure, imprinting, and outcome, multiple measures of imprinting and a more refined analysis linking these endpoints to the exposure and outcome of interest should be included in future studies.

\section{Conclusions}

The precise regulation of imprinted genes is critical for normal development. As imprinting is established early in development, alterations in the environmental milieu during this window can result in aberrations with the potential for deleterious health consequences. The literature does indicate a responsiveness of imprinted genes to environmental factors during specific windows of development, suggesting the potential of these marks to serve as environmental sensors.

Acknowledgments This work was supported by grants from the National Institutes of Health (R01 HD067611/R01ES022223-01A1) and Mount Sinai Children's Environmental Health Center Pilot Fund.

\section{Compliance with Ethics Guidelines}

Conflict of Interest Maya Kappil, Luca Lambertini, and Jia Chen declare that they have no conflict of interest.

Human and Animal Rights and Informed Consent This article does not contain any studies with human or animal subjects performed by any of the authors.

\section{References}

Papers of particular interest, published recently, have been highlighted as:

- Of importance

1. Bartolomei, M. S. \& Ferguson-Smith, A. C. Mammalian genomic imprinting. Cold Spring Harb. Perspect. Biol. 3, (2011).

2. Surani M, Barton S, Norris M. Development of reconstituted mouse eggs suggest imprinting of the genome during gametogenesis. Nature. 1984;308:548-50.

3. McGrath J, Solter D. Completion of mouse embryogenesis requires both the maternal and paternal genomes. Cell. 1984;37:179-83.

4. Renfree MB, Suzuki S, Kaneko-Ishino T. The origin and evolution of genomic imprinting and viviparity in mammals. Philos Trans R Soc Lond B Biol Sci. 2013;368:20120151.

5. Moore T, Haig D. Genomic imprinting in mammalian development: a parental tug-of-war. Trends Genet. 1991;7:45-9.

6. Kinoshita T, Ikeda Y, Ishikawa R. Genomic imprinting: a balance between antagonistic roles of parental chromosomes. Semin Cell Dev Biol. 2008;19:574-9.

7. Kalish JM, Jiang C, Bartolomei MS. Epigenetics and imprinting in human disease. Int J Dev Biol. 2014;58:291-8.

8. Choufani S, Shuman C, Weksberg R. Molecular findings in Beckwith-Wiedemann syndrome. Am J Med Genet C: Semin Med Genet. 2013;163C:131-40.

9. Azzi S, Abi Habib W, Netchine I. Beckwith-Wiedemann and Russell-Silver syndromes: from new molecular insights to the comprehension of imprinting regulation. Curr Opin Endocrinol Diabetes Obes. 2014;21:30-8.

10. Wallace $\mathrm{C}$ et al. The imprinted DLK1-MEG3 gene region on chromosome $14 \mathrm{q} 32.2$ alters susceptibility to type 1 diabetes. Nat Genet. 2010;42:68-71.

11. Kong A et al. Parental origin of sequence variants associated with complex diseases. Nature. 2009;462:868-74.

12. Small KS et al. Identification of an imprinted master trans regulator at the KLF14 locus related to multiple metabolic phenotypes. Nat Genet. 2011;43:561-4. 
13. Talens RP et al. Hypermethylation at loci sensitive to the prenatal environment is associated with increased incidence of myocardial infarction. Int J Epidemiol. 2012;41:106-15.

14. Yuan $J$ et al. Aberrant methylation and silencing of ARHI, an imprinted tumor suppressor gene in which the function is lost in breast cancers. Cancer Res. 2003;63:4174-80.

15. Ito Y et al. Somatically acquired hypomethylation of IGF2 in breast and colorectal cancer. Hum Mol Genet. 2008;17:2633-43.

16. Cui $\mathrm{H}$ et al. Loss of imprinting in colorectal cancer linked to hypomethylation of H19 and IGF2. Cancer Res. 2002;62:6442-6.

17. Nakano $\mathrm{S}$ et al. Expression profile of LIT1/KCNQ1OT1 and epigenetic status at the KvDMR1 in colorectal cancers. Cancer Sci. 2006;97:1147-54.

18. Huang $\mathrm{J}$ et al. Up-regulation of DLK1 as an imprinted gene could contribute to human hepatocellular carcinoma. Carcinogenesis. 2007;28:1094-103.

19. Wu J, Qin Y, Li B, He W, Sun Z. Hypomethylated and hypermethylated profiles of H19DMR are associated with the aberrant imprinting of IGF2 and H19 in human hepatocellular carcinoma. Genomics. 2008;91:443-50.

20. Kuerbitz SJ, Pahys J, Wilson A, Compitello N, Gray TA. Hypermethylation of the imprinted NNAT locus occurs frequently in pediatric acute leukemia. Carcinogenesis. 2002;23:559-64.

21. Feng $\mathrm{W}$ et al. Imprinted tumor suppressor genes ARHI and PEG3 are the most frequently down-regulated in human ovarian cancers by loss of heterozygosity and promoter methylation. Cancer. 2008; 112:1489-502.

22. Brucato N, DeLisi LE, Fisher SE, Francks C. Hypomethylation of the paternally inherited LRRTM1 promoter linked to schizophrenia. Am J Med Genet B Neuropsychiatr Genet. 2014. doi:10.1002/ ajmg.b.32258.

23. Marsit $\mathrm{CJ}$ et al. Placenta-imprinted gene expression association of infant neurobehavior. J Pediatr. 2012;160:854-60. e2.

24. Skaar DA et al. The human imprintome: regulatory mechanisms, methods of ascertainment, and roles in disease susceptibility. ILAR J. 2012;53:341-58.

25. Schulz R et al. Transcript- and tissue-specific imprinting of a tumour suppressor gene. Hum Mol Genet. 2009;18:118-27.

26. Henckel A et al. Histone methylation is mechanistically linked to DNA methylation at imprinting control regions in mammals. Hum Mol Genet. 2009; 18:3375-83.

27. Ooi SKT et al. DNMT3L connects unmethylated lysine 4 of histone H3 to de novo methylation of DNA. Nature. 2007;448:714-7.

28. Luedi PP et al. Computational and experimental identification of novel human imprinted genes. Genome Res. 2007;17:1723-30.

29. Lucifero D, Mann MRW, Bartolomei MS, Trasler JM. Genespecific timing and epigenetic memory in oocyte imprinting. Hum Mol Genet. 2004;13:839-49.

30. Pozharny $\mathrm{Y}$ et al. Genomic loss of imprinting in first-trimester human placenta. Am J Obstet Gynecol. 2010;202:391. e1-8.

31. Court $\mathrm{F}$ et al. Genome-wide parent-of-origin DNA methylation analysis reveals the intricacies of human imprinting and suggests a germline methylation-independent mechanism of establishment. Genome Res. 2014;24:554-69.

32. Santos F, Dean W. Epigenetic reprogramming during early development in mammals. Reproduction. 2004;127:643-51.

33. Kacem, S. \& Feil, R. Chromatin mechanisms in genomic imprinting. Mamm. Genome 20, 544-56.

34. Lee JT. Lessons from X-chromosome inactivation: long ncRNA as guides and tethers to the epigenome. Genes Dev. 2009;23:1831-42.

35. Barlow DP, Bartolomei MS. Genomic imprinting in mammals. Cold Spring Harb Perspect Biol. 2014;6.

36. Chotalia $\mathrm{M}$ et al. Transcription is required for establishment of germline methylation marks at imprinted genes. Genes Dev. 2009;23:105-17.

37. Jirtle, R. L. geneimprint. (2012). at $<$ http://www.geneimprint.com>.
38. Tobi EW et al. DNA methylation differences after exposure to prenatal famine are common and timing- and sex-specific. Hum Mol Genet. 2009;18:4046-53.

39. Williams-Wyss $\mathrm{O}$ et al. Embryo number and periconceptional undernutrition in the sheep have differential effects on adrenal epigenotype, growth, and development. Am J Physiol Endocrinol Metab. 2014;307:E141-50.

40. Obeid R. The metabolic burden of methyl donor deficiency with focus on the betaine homocysteine methyltransferase pathway. Nutrients. 2013;5:3481-95.

41. Haggarty $P$ et al. Folate in pregnancy and imprinted gene and repeat element methylation in the offspring. Am J Clin Nutr. 2013;97:94-9.

42. Stouder C, Somm E, Paoloni-Giacobino A. Prenatal exposure to ethanol: a specific effect on the H19 gene in sperm. Reprod Toxicol. 2011;31:507-12.

43. Liang F et al. Paternal ethanol exposure and behavioral abnormities in offspring: associated alterations in imprinted gene methylation. Neuropharmacology. 2014;81:126-33. While most studies focus on the role of the maternal environment, this study indicates that periconceptional paternal exposures also bear relevance on the imprinting status of the developing fetus.

44. Tyler CR, Allan AM. Prenatal alcohol exposure alters expression of neurogenesis-related genes in an ex vivo cell culture model. Alcohol. 2014;48:483-92.

45. Susiarjo M, Sasson I, Mesaros C, Bartolomei MS. Bisphenol a exposure disrupts genomic imprinting in the mouse. PLoS Genet. 2013;9:e1003401. This study highlights the differential impact of exposures within the embryo and the placenta. Additionally, the study provides a comprehensive evaluation of the exposureoutcome paradigm by including multiple assessments of imprinting, including overall and allele-specific expression and methylation, exploring the impact of the exposure on placenta pathology as a phenotypic outcome, and evaluating the variability in exposure-related susceptibility on imprinting due to the timing of exposure.

46. Kang E-R et al. Effects of endocrine disruptors on imprinted gene expression in the mouse embryo. Epigenetics. 2011;6:937-50.

47. Joubert BR et al. $450 \mathrm{~K}$ epigenome-wide scan identifies differential DNA methylation in newborns related to maternal smoking during pregnancy. Environ Health Perspect. 2012;120:1425-31.

48. Markunas CA et al. Identification of DNA methylation changes in newborns related to maternal smoking during pregnancy. Environ Health Perspect. 2014;122:1147-53.

49. Hiura $\mathrm{H}$ et al. Characterization of DNA methylation errors in patients with imprinting disorders conceived by assisted reproduction technologies. Hum Reprod. 2012;27:2541-8.

50. Nelissen ECM et al. Placentas from pregnancies conceived by IVF/ ICSI have a reduced DNA methylation level at the H19 and MEST differentially methylated regions. Hum Reprod. 2013;28:1117-26.

51. $\mathrm{Xu} \mathrm{X-R} \mathrm{et} \mathrm{al.} \mathrm{Epigenetic} \mathrm{inheritance} \mathrm{of} \mathrm{paternally} \mathrm{expressed}$ imprinted genes in the testes of ICSI mice. Curr Pharm Des. 2014;20:1764-71.

52. Lambertini $\mathrm{L}$ et al. A sensitive functional assay reveals frequent loss of genomic imprinting in human placenta. Epigenetics. 2008;3: 261-9.

53. Lambertini L et al. NIH Public Access. 2009;3:261-9.

54. Monk D et al. Limited evolutionary conservation of imprinting in the human placenta. Proc Natl Acad Sci U S A. 2006;103:6623-8.

55. Brideau CM, Eilertson KE, Hagarman JA, Bustamante CD, Soloway PD. Successful computational prediction of novel imprinted genes from epigenomic features. Mol Cell Biol. 2010;30:3357-70.

56. Toso L, Roberson R, Woodard J, Abebe D, Spong CY. Prenatal alcohol exposure alters GABA(A)alpha5 expression: a mechanism of alcohol-induced learning dysfunction. Am J Obstet Gynecol. 2006;195:522-7. 
57. Krishnamoorthy M, Gerwe BA, Scharer CD, Heimburg-Molinaro J, Gregory F, Nash RJ, et al. GABRB3 gene expression increases upon ethanol exposure in human embryonic stem cells. J Recept Signal Transduct Res. 2011;31:206-13.

58. Zhang XF, Zhang LJ, Feng YN, Chen B, Feng YM, Liang GJ, et al. Bisphenol A exposure modifies DNA methylation of imprint genes in mouse fetal germ cells. Mol Biol Rep. 2012;39:8621-8.

59. Vucetic Z, Totoki K, Schoch H, Whitaker KW, Hill-Smith T, Lucki I, et al. Early life protein restriction alters dopamine circuitry. Neuroscience. 2010;168:359-70.

60. Ba Y, Yu H, Liu F, Geng X, Zhu C, Zhu Q, et al. Relationship of folate, vitamin B12 and methylation of insulin-like growth factor-II in maternal and cord blood. Eur J Clin Nutr. 2011;65:480-5.
61. Tobi EW, Slagboom PE, van Dongen J, Kremer D, Stein AD, Putter $\mathrm{H}$, et al. Prenatal famine and genetic variation are independently and additively associated with DNA methylation at regulatory loci within IGF2/H19. PLoS One. 2012;7:e37933.

62. Houde AA, Guay SP, Desgagné V, Hivert MF, Baillargeon JP, StPierre J, et al. Adaptations of placental and cord blood ABCA1 DNA methylation profile to maternal metabolic status. Epigenetics. 2013;8:1289-302.

63. Hoyo C, Daltveit AK, Iversen E, Benjamin-Neelon SE, Fuemmeler B, Schildkraut J, et al. Erythrocyte folate concentrations, CpG methylation at genomically imprinted domains, and birth weight in a multiethnic newborn cohort. Epigenetics. 2014;9:1120-30. 